\title{
Synthesis and Some Properties of New 5-Hydroxy-2-[(hetarylthio)methyl]-4H-pyran-4-ones
}

\author{
V. V. Dotsenko ${ }^{a, b, *}$, D. D. Guz ${ }^{a}$, D. T. Tebiev ${ }^{a}$, V. K. Kindop ${ }^{a}$, N. A. Aksenov ${ }^{b}$, \\ I. V. Aksenova ${ }^{b}$, and E. E. Netreba ${ }^{c}$ \\ ${ }^{a}$ Kuban State University, Krasnodar, 350040 Russia \\ ${ }^{b}$ North Caucasus Federal University, Stavropol, 355009 Russia \\ ${ }^{c}$ Taurida Academy of the V.I. Vernadsky Crimean Federal University, Simferopol, 295007 Russia \\ *e-mail:victor_dotsenko_@mail.ru
}

Received July 10, 2021; revised July 28, 2021; accepted July 29, 2021

\begin{abstract}
The reaction of 2-thioxoazines with chlorokojic acid in the presence of KOH in DMF led to the formation of new hybrid molecules containing fragments of kojic acid and azaheterocycle linked by the $\mathrm{SCH}_{2}$ spacer. In silico prediction of bioavailability parameters was carried out, possible protein targets were predicted by the protein ligand docking method.
\end{abstract}

Keywords: chlorokojic acid, 3-cyanopyridine-2(1H)-thiones, 2-thioxo-1,2-dihydroquinoxalines, $S$-alkylation, calculated biological activity

DOI: $10.1134 / \mathrm{S} 107036322109005 \mathrm{X}$

Kojic acid 1 (5-hydroxy-2-hydroxymethyl-4H-pyran4-one) is widely used in the pharmaceutical industry, agrochemistry, cosmetology [1-5], and as a ligand for complex compounds [6] and building blocks for construction of biologically active heterocyclic molecules [7-9]. Being one of the most studied and available non-toxic tyrosinase inhibitors [10,11], kojic acid as a bioactive molecule is not devoid of disadvantages, among which should be noted the lack of stability during storage and a relatively low inhibitory activity. For this reason, in recent years, the direction of the $\gamma$-pyrones chemistry devoted to the preparation of conjugates of kojic acid or other derivatives functionalized at $\mathrm{C}^{6} \mathrm{OH}$ groups has been developed [12-18]. Among the new effective tyrosinase inhibitors, it is worth noting a number of hybrid molecules combining a kojic acid residue and an aryl/hetaryl moiety - for example, Mannich base 2 [18], 1,2,3-triazole derivatives 3 [19, 20], substituted thiophenol 4 [21] or 4-amino-1,2,4-triazoles 5 [22] (Scheme 1). In addition, a number of hybrid structures with a kojic acid motif are known, demonstrating a different spectrum of biological action. Thus, functionalization with a kojic acid was used to create ${ }^{10} \mathrm{~B}$-labeled derivatives of dodecaboranethiol
6 for boron neutron capture therapy of cancer [23]. Compound ML221 7 is a highly effective antagonist of the APJ apelin receptor with possible use in the therapy of cardiovascular diseases [24] (Scheme 1). Pivalic acid esters 8 have been shown to be effective inhibitors of neutrophil elastase suitable for the treatment of inflammatory lung diseases [25], while piperazine derivatives 9 exhibit antituberculosis [26] and anticancer [27] effects. According to patent data [28], 2-mercaptoimidazoline derivatives 10 exhibit antibacterial activity.

One of the most accessible methods of functionalization of kojic acid is its transformation into bromo- or chlorokojic acid 11, with further replacement of the halogen atom by various nucleophiles such as thioureas [28-33], alkyl mercaptans [29, 30, 34], thiophenols $[21,35]$, sodium arylsulfinates [36], alkali metal thiocyanates [36-39], $S$-glycosylisothiuronium salts [40], various mercaptoazoles [22, 25, 28, 29, 41], 2-mercaptopyrimidines [24, 42], 2-mercaptoquinazolines $[43,44]$, mono- and dithiophosphates [45, 46]. Many of the compounds obtained in this way are of interest for agrochemistry as growth regulators and herbicides comparable in efficiency to Fluazifop-P [29], or 
Scheme 1.<smiles>O=c1cc(CO)occ1O</smiles>

Kojic acid, 1<smiles>O=c1cc(CN2CCCC2)oc(CN2CCN(CC3CCCCC3)CC2)c1O</smiles>

2<smiles>O=c1cc(Cn2cc(CO[Al])nn2)occ1O</smiles>

3<smiles>O=c1cc(CSc2ccc(O)cc2)occ1O</smiles>

4<smiles>[R]c1nnc(SCc2cc(=O)c(O)co2)n1N</smiles>

5

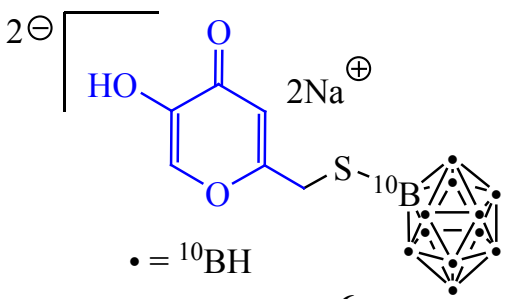

6<smiles></smiles><smiles>[R]c1ccc2nc(SCc3cc(=O)c(OC(=O)C(C)(C)C)co3)[X]c2c1[R]=O</smiles>
$\mathbf{8}(\mathrm{X}=\mathrm{O}, \mathrm{S})$<smiles>[X]Cc1cc(=O)c(O)c(CN2CCN([R])CC2)o1</smiles><smiles>[R]Oc1coc(CSC2=NCCN2)cc1=O</smiles><smiles>O=c1cc(C[Hg])occ1O</smiles>

as insecticides $[45,46]$. In general, the analysis of literature data allows us to conclude that the reaction of halokojic acids with heterocyclic $S$-nucleophiles has been relatively little studied. For example, the reaction of 5-hydroxy-2-chloromethyl-4H-pyran-4-one with available 3 -cyanopyridine-2(1H)-thiones and tautomeric mercaptans (for review works, see [47-54]) widely used in heterocyclic synthesis have not been reported earlier. At the same time, the target products can be of interest as promising pharmaceuticals, agrochemicals and reagents for fine organic synthesis.

We performed the reaction of some active $S$-nucleophiles of the azaheterocyclic series with chlorkojic acid $\mathbf{1 1}(\mathrm{Hlg}=\mathrm{Cl})$. It was found that 2-thioxopyridines $\mathbf{1 2 a}, \mathbf{1 2 b}$ react with chloride $\mathbf{1 1}$ in the presence of 1 equiv. of $10 \%$ aqueous $\mathrm{KOH}$ with the formation of previously unknown hybrid molecules 13a, 13b containing kojic acid and nicotinonitrile fragments (Scheme 2). Under similar conditions, 2-thioxo-1,2dihydroquinoxaline $\mathbf{1 4}$ was converted to compound $\mathbf{1 5}$ in $28 \%$ yield.

Structure of the obtained compounds was confirmed by a complex of spectral data. The IR spectra of compounds 13 and 15 show an absorption band at $1646-1649 \mathrm{~cm}^{-1}$, corresponding to stretching vibrations of the conjugated carbonyl group of $\gamma$-pyrone, as well as a broad band at $3226-3253 \mathrm{~cm}^{-1}(\mathrm{O}-\mathrm{H})$. The spectra of compounds 13a, 13b also show an absorption band in the range of $2218-2223 \mathrm{~cm}^{-1}$ (conjugated cyano group). The ${ }^{1} \mathrm{H}$ NMR spectra of compounds $\mathbf{1 3}$ and $\mathbf{1 5}$ exhibit singlets at 4.47-4.64 ppm $\left(\mathrm{SCH}_{2}\right)$, as well as signals of the 5-hydroxypyran-4-one fragment at 6.47-6.59 $\left(\mathrm{H}^{3}\right)$, 8.01-8.09 $\left(\mathrm{H}^{6}\right)$, and 9.11-9.15 ppm $(\mathrm{OH})$. The ${ }^{13} \mathrm{C}$ NMR spectra of compounds $\mathbf{1 3}$ and $\mathbf{1 5}$ contain characteristic 
Scheme 2.<smiles>N#Cc1c(Br)cc(-c2ccccc2)[nH]c1=S</smiles>

12a, 12b<smiles>O=c1cc(CCl)occ1O</smiles>

11

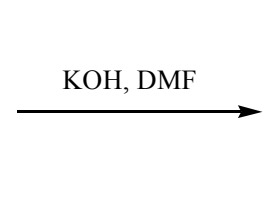

$\overbrace{\mathrm{S}}^{\mathrm{OCH}_{3}}$

14<smiles>O=c1cc(CCl)occ1O</smiles>

11<smiles></smiles>

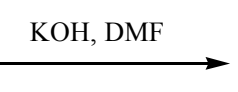<smiles>COc1ccc(-c2nc3ccccc3nc2SCc2cc(=O)c(O)co2)cc1</smiles>

$\mathrm{Ar}=\mathrm{Ph}(\mathbf{a}), 2,4-\mathrm{Cl}_{2} \mathrm{C}_{6} \mathrm{H}_{3}(\mathbf{b})$.

signals at 30.8-31.6 $\left(\mathrm{SCH}_{2}\right), 112.1-112.9\left(\mathrm{C}^{3}\right.$-pyran $)$, 139.6-139.8 (C6 -pyran), 143.5-145.8 ( $\mathrm{C}^{5}$-pyran $), 163.1-$ $163.8\left(\mathrm{C}^{2}\right.$-pyran $)$, and $173.6 \mathrm{ppm}(\mathrm{C}=\mathrm{O})$.

In the context of the known biological activity of kojic acid derivatives [7-11], nicotinonitriles, and quinoxalines (for recent reviews see [55-58] and [59-67], respectively), it seemed appropriate to perform a preliminary in silico study of possible targets, ADMET parameters, and the bioavailability criteria for new hybrid molecules. Analysis of the structures for compliance with the "Lipinski's rule of five" [molecule mass (MW) $\leq$ $500, c \log P \leq 5.0$, TPSA $\leq 140 \AA^{2}$, number of hydrogen bond acceptors $\leq 10$, donors $\leq 5]$ [68-70] was carried out using the OSIRIS Property Explorer software service [71]. The following parameters were calculated: $c \log P$ (logarithm of the partition coefficient between $n$-octanol and water $\log \left(c_{\text {octanol }} / c_{\text {water }}\right)$, solubility $(\log S)$, Topological Polar Surface Area (TPSA), a number of toxicological characteristics such as risks of side effects (mutagenic, oncogenic, reproductive effects), the druglikeness parameter, as well as the overall assessment of the pharmacological potential of the compound (drug score). The calculated data are presented in Table 1.

The $c \log P$ value for all the studied structures indicates probable good absorption and permeability [68-70]. At the same time, for all the compounds, the $\log S$ value $<-4.0$ indicates low solubility (less than $\left.1 \times 10^{-4} \mathrm{~mol} / \mathrm{L}\right)$. The molecular weights of all the compounds and the TPSA parameter met the criteria for oral bioavailability. The studied compounds show a moderate risk of oncogenic action associated with the presence of a 5-hydroxypyran-4-one fragment. However, the total predicted values of the drug score are rather high. To predict the ADMET parameters (Absorption, Distribution, Metabolism, Excretion, Toxicity) and probable targets, the SwissADME [72] and GUSAR [73] software packages were also used. The results are shown in Table 2. In general, the assessment of acute toxicity

Table 1. Toxicity risks and physico-chemical parameters of compounds $\mathbf{1 3 a}, \mathbf{1 3 b}, \mathbf{1 5}$ predicted by OSIRIS Property Explorer

\begin{tabular}{c|c|c|c|c|c|c|c|c|c|c}
\hline \multirow{2}{*}{ Compound } & \multicolumn{4}{|c|}{ Toxicity risk $^{\mathrm{a}}$} & \multicolumn{6}{c}{ Physico-chemical parameters } \\
\cline { 2 - 11 } & $\mathrm{A}$ & $\mathrm{B}$ & $\mathrm{C}$ & $\mathrm{D}$ & $c \log P$ & $\log S$ & $\mathrm{MW}$ & TPSA & $\begin{array}{c}\text { drug- } \\
\text { likeness }\end{array}$ & $\begin{array}{c}\text { drug } \\
\text { score }\end{array}$ \\
\hline $\mathbf{1 3 a}$ & - & \pm & - & - & 3.81 & -6.86 & 412 & 108.5 & -2.83 & 0.18 \\
$\mathbf{1 3 b}$ & - & \pm & - & - & 5.02 & -8.34 & 480 & 108.5 & -1.53 & 0.14 \\
$\mathbf{1 5}$ & + & \pm & - & + & 2.69 & -4.62 & 392 & 106.8 & 1.93 & 0.31 \\
\hline
\end{tabular}

a The sign "+" indicates a high risk of toxicity, " \pm " - a moderate risk, " $"$ — no toxicity. A-Mutagenicity, B-carcinogenicity, C-irritant effect, D-reproductive effects. 
Table 2. Calculated ADMET parameters for compounds $\mathbf{1 3 a}, \mathbf{1 3 b}$, and $\mathbf{1 5}^{\mathrm{a}}$

\begin{tabular}{|c|c|c|c|c|c|c|c|c|c|c|}
\hline \multirow[b]{2}{*}{ Comp. no. } & \multirow[b]{2}{*}{$\begin{array}{l}\text { Penetration } \\
\text { through BBB }\end{array}$} & \multirow[b]{2}{*}{$\begin{array}{l}\text { Gastrointestinal } \\
\text { absorption }\end{array}$} & \multicolumn{5}{|c|}{$\begin{array}{l}\text { Inhibition of cytochromes } \\
\text { P450 }\end{array}$} & \multicolumn{3}{|c|}{$\begin{array}{l}\text { Acute toxicity (rats), } \mathrm{LD}_{50} \\
{[\log (\mathrm{mmol} / \mathrm{kg}) /(\mathrm{mg} / \mathrm{kg})]^{\mathrm{b}}}\end{array}$} \\
\hline & & & $\frac{2}{2}$ & 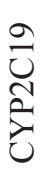 & 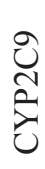 & 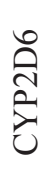 & 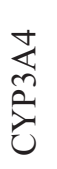 & IP & IV & Oral \\
\hline $13 a$ & - & + & + & + & + & + & + & $\frac{0.215}{677.4}$ & $\frac{-0.429}{153.7}$ & $\frac{0.472}{1224.0}$ \\
\hline $13 b$ & - & - & + & + & + & - & + & $\frac{0.167}{706.5}$ & $\frac{-0.484}{157.8}$ & $\frac{0.728}{2571.0}$ \\
\hline 15 & - & + & + & + & + & + & + & $\frac{-0.007}{385.9}$ & $\frac{-0.423}{148.2}$ & $\frac{0.716}{2040.0}$ \\
\hline
\end{tabular}

a The sign "+" or "_" indicates the presence or absence of the effect.

${ }^{\mathrm{b}}$ IP (IntraPeritoneal) — intraperitoneal administration, IV (IntraVenous)—intravenous administration, Oral—oral administration.

allows all the studied compounds to be classified as IV and V hazard classes according to the OECD criteria [74]. For these compounds, an inhibitory effect on a wide range of cytochrome $\mathrm{P} 450$ isoforms is postulated.

Possible protein targets for the obtained compounds were predicted using the new Galaxy Sagittarius protein ligand docking protocol [75] based on the GalaxyWeb web server $[76,77]$. The 3D joint structures were preoptimized by molecular mechanics in an MM2 force field to optimize geometry and minimize energy. Docking using the GalaxySagittarius protocol was performed in the Binding compatability prediction and Re-ranking using docking modes. Table 3 shows the results of docking for each of compounds 13a, 13b, 15 for 10 target-ligand complexes with the minimum free binding energy $\Delta G_{\text {bind }}$ and the best estimate of the protein-ligand interaction. Predicted protein targets are specified using ID-identifiers in the Protein Data Bank (PDB) and in the UniProt database. As one can see from the Table 3, common receptors for compounds $\mathbf{1 3 a}, \mathbf{1 3 b}$, and $\mathbf{1 5}$ are RPA (Replication Protein A, PDB ID 4luv) phosphoprotein responsible for DNA replication and repair in eukaryotes, 3-phosphoinositol-dependent protein kinase-1 (PDK1, PDB ID 4rqv), and apoptosis regulator Mcl-1 (PDB ID 6qfq) (Fig. 1). Thus, 5-hydroxy-2-[(hetarylthio)methyl]4H-pyran-4-ones 13a, 13b, and $\mathbf{1 5}$ can be considered as promising objects for screening in order to search for new agents for the treatment and therapy of oncological diseases.
In conclusion, we developed a convenient method for the preparation of previously unknown 5-hydroxy2-[(hetarylthio)methyl]-4H-pyran-4-ones by the reaction of 2-thioxonicotinonitrile and 2-thioxo-1,2dihydroquinoxaline derivatives with 5-hydroxy-2chloromethyl-4H-pyran-4-one (chlorokojic acid). The results of in silico experiments on the assessment of probable protein targets, toxicity, and bioavailability parameters make it possible to consider the obtained compounds as promising objects for the development of new drugs with antitumor action.

\section{EXPERIMENTAL}

IR spectra were registered on a Bruker Vertex 70 spectrometer with an ATR attachment. ${ }^{1} \mathrm{H}$ and ${ }^{13} \mathrm{C}$ NMR spectra were recorded on a Bruker Avance III HD $400 \mathrm{MHz}$ instrument (400.17 and $100.63 \mathrm{MHz}$, respectively) in a DMSO- $d_{6}$ solution. Chromato-mass spectra were recorded on a Bruker Customer MicrOTOF instrument in the range of $m / z 50-1200$, the method of ionization was electrospray (ESI). Elemental analysis was performed on an Elementar vario Micro cube instrument. The individuality of the obtained samples was controlled by TLC on Sorbfil-A plates (LLC Imid, Krasnodar), eluent was acetone-hexane mixture $(1: 1)$ or ethyl acetate, visualized with iodine vapors or UV light.

The starting 3-cyanopyridine-2(1H)-thiones 12a, 12b [80, 81] and 2-thioxo-1,2-dihydroquinoxaline $\mathbf{1 4}$ [82] were obtained according to the known methods. 
Table 3. Results of prediction of protein-ligand interaction for compounds $\mathbf{1 3 a}, \mathbf{1 3 b}, \mathbf{1 5}$

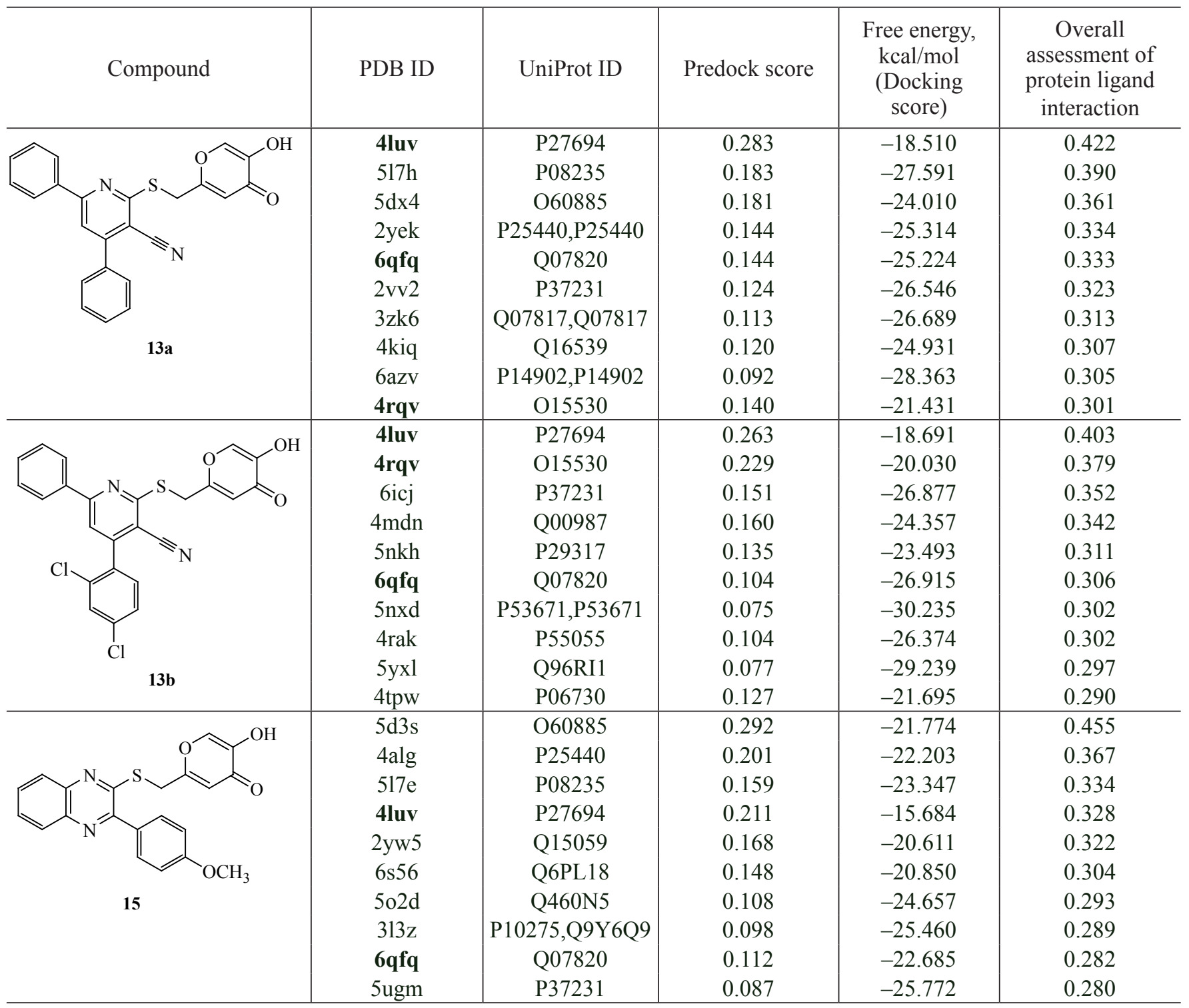

Chlorokojic acid $\mathbf{1 1}$ was prepared by treating commercial kojic acid with thionyl chloride [83].

General procedure for the synthesis of 5-hydroxy2-[(hetarylthio)methyl]-4H-pyran-4-ones 13a, 13b, 15. The appropriate 2-thioxonicotinonitrile 12 or thione $14(2 \mathrm{mmol})$ was suspended in $2 \mathrm{~mL}$ of DMF, then an aqueous $10 \% \mathrm{KOH}$ solution $(1.03 \mathrm{~mL}, 2 \mathrm{mmol}$, $d 1.09 \mathrm{~g} / \mathrm{mL}$ ) was added. The resulting suspension was stirred at room temperature until dissolved, then through a paper filter it was added dropwise to a solution of $321 \mathrm{mg}$ ( $2 \mathrm{mmol}$ ) of chlorokojic acid 11 in $0.5 \mathrm{~mL}$ of DMF. The resulting mixture was stirred for $30 \mathrm{~min}$. The formed precipitate was filtered off, washed with $50 \%$ ethanol, and dried at $60^{\circ} \mathrm{C}$.

2-\{[(5-Hydroxy-4-oxo-4H-pyran-2-yl)methyl]thio\}-4,6-diphenylnicotinonitrile (13a). Yield 75\%, pale brown powder. IR spectrum, $v, \mathrm{~cm}^{-1}: 1647(\mathrm{C}=\mathrm{O}), 2218$ $(\mathrm{C} \equiv \mathrm{N}), 3239(\mathrm{O}-\mathrm{H}) .{ }^{1} \mathrm{H}$ NMR spectrum, $\delta$, ppm: $4.64 \mathrm{~s}$ $\left(2 \mathrm{H}, \mathrm{SCH}_{2}\right), 6.50 \mathrm{~s}\left(1 \mathrm{H}, \mathrm{H}^{3}{ }_{\text {pyran }}\right), 7.53-7.59 \mathrm{~m}(6 \mathrm{H}, \mathrm{H}-\mathrm{Ph})$, 7.74-7.77 m (2H, H-Ph), $7.96 \mathrm{~s}\left(1 \mathrm{H}, \mathrm{H}^{5}-\mathrm{Py}\right), 8.09 \mathrm{~s}(1 \mathrm{H}$, $\left.\mathrm{H}^{6}{ }_{\text {pyran }}\right), 8.24-8.26 \mathrm{~m}(2 \mathrm{H}, \mathrm{H}-\mathrm{Ph}), 9.15$ br. s $(1 \mathrm{H}, \mathrm{OH})$. ${ }^{13} \mathrm{C}$ DEPTQ NMR spectrum, $\delta_{\mathrm{C}}$, ppm: $30.8\left(\mathrm{SCH}_{2}\right), 103.1$ $(\underline{\mathrm{C}}-\mathrm{C} \equiv \mathrm{N}), 112.2^{*}\left(\mathrm{C}^{3} \mathrm{H}\right.$, pyran $), 115.5(\mathrm{C} \equiv \mathrm{N}), 116.9^{*}\left(\mathrm{C}^{5}\right.$, Py), 127.6* (2C, CH, Ph), 128.7* (2C, CH, Ph), 128.9* (2C, $\mathrm{CH}, \mathrm{Ph}), 129.0^{*}(2 \mathrm{C}, \mathrm{CH}, \mathrm{Ph}), 130.2^{*}\left(\mathrm{C}^{4} \mathrm{H}, \mathrm{Ph}\right)$, 
(a)

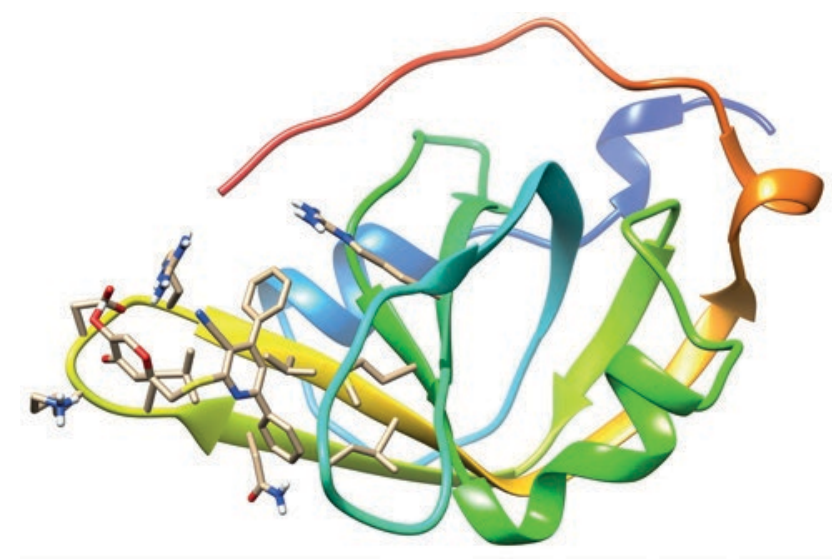

(c)

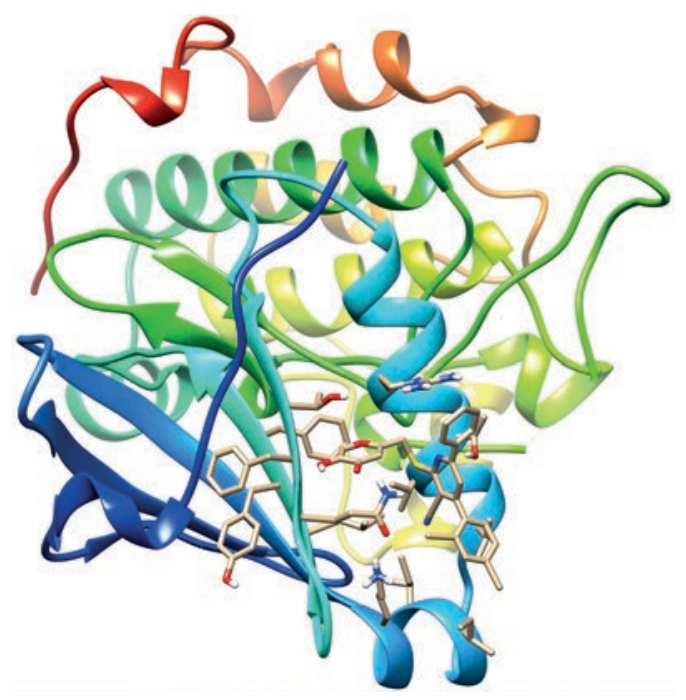

(b)

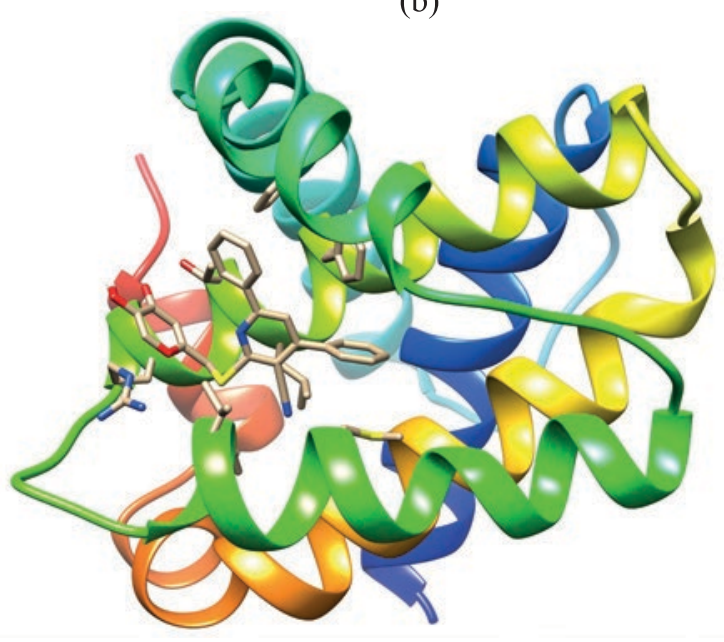

(d)

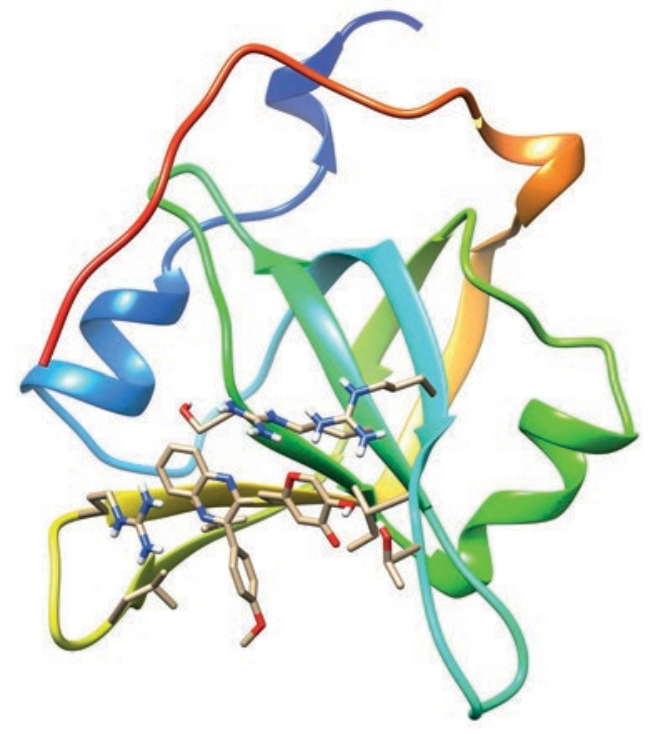

Fig. 1. Predicted structures of protein-ligand complexes for compound 13a and phosphoprotein RPA (PDB ID 4luv) (a), compound 13a and protein Mcl-1 (PDB ID 6qfq) (b), compound 13b and protein kinase PDK1 (PDB ID 4rqv) (c), quinoxaline 15 and phosphoprotein RPA (PDB ID 4luv) (d) (obtained using the Galaxy Sagittarius protocol). Molecular graphics were visualized using the UCSF Chimera software package $[78,79]$.

131.0* ( $\left.\mathrm{C}^{4} \mathrm{H}, \mathrm{Ph}\right), 135.5\left(\mathrm{C}^{1}, \mathrm{Ph}\right), 136.4\left(\mathrm{C}^{1}, \mathrm{Ph}\right), 139.8^{*}$ ( ${ }^{6} \mathrm{H}$, pyran), $145.8\left(\mathrm{C}^{5}\right.$, pyran), $154.5(\mathrm{Py}), 158.1$ (Py), $160.6(\mathrm{Py}), 163.8\left(\mathrm{C}^{2}\right.$, pyran $), 173.6(\mathrm{C}=\mathrm{O})$. Hereinafter, an asterisk denotes signals in antiphase. Mass spectrum, $m / z\left(I_{\text {rel }}, \%\right): 451.05[M+\mathrm{K}]^{+}(100), 863.15[2 M+$ $\mathrm{K}]^{+}$. Found, \%: C 69.77; H 4.06; N 6.90. $\mathrm{C}_{24} \mathrm{H}_{16} \mathrm{~N}_{2} \mathrm{O}_{3} \mathrm{~S}$ (M 412.46). Calculated, \%: C 69.89; H 3.91; N 6.79.

2-\{[(5-Hydroxy-4-oxo-4H-pyran-2-yl)methyl]thio\}6-phenyl-4-(2,4-dichlorophenyl)nicotinonitrile (13b). Yield 30\%, brown powder. IR spectrum, $v, \mathrm{~cm}^{-1}: 1649$
$(\mathrm{C}=\mathrm{O}), 2223(\mathrm{C} \equiv \mathrm{N}), 3226(\mathrm{O}-\mathrm{H}) .{ }^{1} \mathrm{H}$ NMR spectrum, $\delta$, ppm: $4.64 \mathrm{~s}\left(2 \mathrm{H}, \mathrm{SCH}_{2}\right), 6.47 \mathrm{~s}\left(1 \mathrm{H}, \mathrm{H}^{3}{ }_{\text {pyran }}\right), 7.52-7.56 \mathrm{~m}$ (5H, H-Ar), 7.65-7.66 m (2H, H-Ar), $7.90 \mathrm{~d}(1 \mathrm{H}, \mathrm{H}-\mathrm{Ar}$, ${ }^{4} J 0.9$ Гц), $7.99 \mathrm{~s}\left(1 \mathrm{H}, \mathrm{H}^{5}-\mathrm{Py}\right), 8.01 \mathrm{~s}\left(1 \mathrm{H}, \mathrm{H}_{\text {pyran }}^{6}\right)$, 8.22-8.24 m (2H, H-Ar). Signal of the $\mathrm{OH}$ group does not appear due to deuterium exchange. ${ }^{13} \mathrm{C}$ DEPTQ NMR spectrum, $\delta_{\mathrm{C}}, \mathrm{ppm}: 30.8\left(\mathrm{SCH}_{2}\right), 104.6(\underline{\mathrm{C}}-\mathrm{C} \equiv \mathrm{N}), 112.1^{*}$ $\left(\mathrm{C}^{3} \mathrm{H}\right.$, pyran $), 114.5(\mathrm{C} \equiv \mathrm{N}), 117.5^{*}\left(\mathrm{C}^{5}, \mathrm{Py}\right), 127.6^{*}(\mathrm{CH}$, $\mathrm{Ar}), 127.9 *(\mathrm{CH}, \mathrm{Ar}), 129.1 *(\mathrm{CH}, \mathrm{Ar}), 131.2 *(\mathrm{CH}, \mathrm{Ar})$, 132.3* (CH, Ar), 132.5 (Ar), 133.6 (Ar), 135.4 (Ar), 136.1 
(Ar), 139.6* (C ${ }^{6} \mathrm{H}$, pyran), $145.8\left(\mathrm{C}^{5}\right.$, pyran), 151.5 (Py), 158.3 (Py), 160.1 (Py), $163.1\left(\mathrm{C}^{2}\right.$, pyran), $173.6(\mathrm{C}=\mathrm{O})$. Mass spectrum, $m / z\left(I_{\text {rel }}, \%\right): 481.50[M+\mathrm{Na}]^{+}(100)$, $985.02[2 M+\mathrm{Na}]^{+}$. Found, \%: C 59.74; H 3.10; N 5.94. $\mathrm{C}_{24} \mathrm{H}_{14} \mathrm{Cl}_{2} \mathrm{~N}_{2} \mathrm{O}_{3} \mathrm{~S}$ ( $M$ 481.35). Calculated, \%: C 59.89; $\mathrm{H} 2.93$; N 5.82 .

\section{5-Hydroxy-2-(\{[3-(4-methoxyphenyl)quinoxalin-} 2-yl]thio\} methyl)-4H-pyran-4-one (15). Yield 28\%, brown powder. IR spectrum, $v, \mathrm{~cm}^{-1}: 1646(\mathrm{C}=\mathrm{O}), 3253$ (O-H). ${ }^{1} \mathrm{H}$ NMR spectrum, $\delta$, ppm: $3.85 \mathrm{~s}(3 \mathrm{H}, \mathrm{MeO})$, $4.47 \mathrm{~s}\left(2 \mathrm{H}, \mathrm{SCH}_{2}\right), 6.59 \mathrm{~s}\left(1 \mathrm{H}, \mathrm{H}_{\text {pyran }}^{3}\right), 7.10-7.12 \mathrm{~m}(2 \mathrm{H}$, $\mathrm{H}-\mathrm{Ar}), 7.72-8.03 \mathrm{~m}(8 \mathrm{H}, \mathrm{H}-\mathrm{Ar}$, overlapping with the signal of $\left.\mathrm{H}_{\text {pyran }}^{5}\right), 9.11 \mathrm{~s}(1 \mathrm{H}, \mathrm{OH}) .{ }^{13} \mathrm{C}$ DEPTQ NMR spectrum, $\delta_{\mathrm{C}}$, ppm: $31.6\left(\mathrm{SCH}_{2}\right), 55.3 *(\mathrm{MeO}), 112.9 *$ ( $\mathrm{C}^{3} \mathrm{H}$, pyran $), 113.9^{*}(\mathrm{CH}, \mathrm{Ar}), 127.1^{*}(\mathrm{CH}, \mathrm{Ar}), 128.8^{*}$ $(\mathrm{CH}, \mathrm{Ar}), 129.0 *(\mathrm{CH}, \mathrm{Ar}), 129,1 *(\mathrm{CH}, \mathrm{Ar}), 130.4 *(\mathrm{CH}$, $\mathrm{Ar}), 130.5^{*}(\mathrm{CH}, \mathrm{Ar}), 139.1$ (Ar), 139.8* (CH, pyran), 143.5* (C $\mathrm{C}^{5}$, pyran), 145.7 (Ar), $152.4(\mathrm{Ar}), 152.9(\mathrm{Ar})$, 160.6 (Ar), $163.6\left(\mathrm{C}^{2}\right.$, pyran), $173.6(\mathrm{C}=\mathrm{O})$.. Found, \%: C 64.15; H 4.22; N 7.24. $\mathrm{C}_{21} \mathrm{H}_{16} \mathrm{~N}_{2} \mathrm{O}_{4} \mathrm{~S}(M 392.43)$. Calculated, \%: C 64.27; H 4.11; N 7.14.

\section{AUTHOR INFORMATION}

V.V. Dotsenko, ORCID: https://orcid.org/0000-0001-71630497

D.D. Guz, ORCID: https://orcid.org/0000-0003-1230-1305

D.T. Tebiev, ORCID: https://orcid.org/0000-0003-28552205

V.K. Kindop, ORCID: https://orcid.org/0000-0002-90059668

N.A. Aksenov, ORCID: https://orcid.org/0000-0002-71259066

I.V. Aksenova, ORCID: https://orcid.org/0000-0002-80831407

E.E. Netreba, ORCID: https://orcid.org/0000-0001-92921904

\section{ACKNOWLEDGMENTS}

The studies were carried out using the equipment of the Research and Education Center "Diagnostics of the Structure and Properties of Nanomaterials" and the Ecological Analytical Center of the Kuban State University.

\section{FUNDING}

This work was financially supported by the Russian Foundation for Basic Research and the Krasnodar Territory Administration (project no. 19-43-230007 r_a), as well as by the
Ministry of Education and Science of the Russian Federation (topic 0795-2020-0031).

\section{CONFLICT OF INTEREST}

No conflict of interest was declared by the authors.

\section{OPEN ACCESS}

This article is licensed under a Creative Commons Attribution 4.0 International License, which permits use, sharing, adaptation, distribution and reproduction in any medium or format, as long as you give appropriate credit to the original author(s) and the source, provide a link to the Creative Commons license, and indicate if changes were made. The images or other third party material in this article are included in the article's Creative Commons license, unless indicated otherwise in a credit line to the material. If material is not included in the article's Creative Commons license and your intended use is not permitted by statutory regulation or exceeds the permitted use, you will need to obtain permission directly from the copyright holder. To view a copy of this license, visit http://creativecommons.org/licenses/by/4.0/.

\section{REFERENCES}

1. Beélik, A., Adv. Carbohyd. Chem., 1956, vol. 11, p. 145 . https://doi.org/10.1016/S0096-5332(08)60118-6

2. Saeedi, M., Eslamifar, M., and Khezri, K., Biomed. Pharmacother., 2019, vol. 110, p. 582. https://doi.org/10.1016/j.biopha.2018.12.006

3. Aytemir, M.D. and Karakaya, G., Kojic Acid Derivatives. Medicinal Chemistry and Drug Design, Rijeka: InTech Open Access Publisher, 2012, p. 1. https://doi.org/10.5772/31006

4. Chaudhary, J., Pathak, A.N., and Lakhawat, S., Ann. Res. Rev. Biol., 2014, p. 3165.

https://doi.org/10.9734/ARRB/2014/10643

5. Mohamad, R., Mohamed, M.S., Suhaili, N., Salleh, M.M., and Ariff, A.B., Biotech. Mol. Biol. Rev., 2010, vol. 5, no. 2, p. 24. https://doi.org/10.5897/BMBR2010.0004

6. Kandioller, W., Kurzwernhart, A., Hanif, M., Meier, S.M., Henke, H., Keppler, B.K., and Hartinger, C.G., J. Organomet. Chem., 2011, vol. 696, no. 5, p. 999.

https://doi.org/10.1016/j.jorganchem.2010.11.010

7. Zirak, M. and Eftekhari-Sis, B., Turk. J. Chem., 2015, vol. 39 , no. 3, p. 439. https://doi.org/10.3906/kim-1502-55

8. Mohajer, F. and Mohammadi Ziarani, G., Heterocycles, 
2021, vol. 102, no. 2, p. 211.

https://doi.org/10.3987/REV-20-936

9. Chaudhary, A., Curr. Org. Chem., 2020, vol. 24, no. 14, p. 1643.

https://doi.org/10.2174/1385272824999200622113153

10. Saruno, R., Kato, F., and Ikeno, T., Agric. Biol. Chem., 1979, vol. 43, no. 6, p. 1337.

https://doi.org/10.1271/bbb1961.43.1337

11. Cabanes, J., Chazarra, S., and Garcia-Carmona, F., J. Pharm. Pharmacol., 1994, vol. 46, no. 12, p. 982. https://doi.org/10.1111/j.2042-7158.1994.tb03253.x

12. Noh, J.M., Kwak, S.Y., Kim, D.H., and Lee, Y.S., Biopolymers (Pept. Sci.), 2007, vol. 88, no. 2, p. 300. https://doi.org/10.1002/bip.20670

13. Noh, J.M., Kwak, S.Y., Seo, H.S., Seo, J.H., Kim, B.G., and Lee, Y.S., Bioorg. Med. Chem. Lett., 2009, vol. 19 , no. 19 , p. 5586.

https://doi.org/10.1016/j.bmcl.2009.08.041

14. Lee, Y.S., Park, J.H., Kim, M.H., Seo, S.H., and Kim, H.J., Arch. Pharm., 2006, vol. 339, no. 3, p. 111. https://doi.org/10.1002/ardp.200500213

15. Singh, B.K., Park, S.H., Lee, H.B., Goo, Y.A., Kim, H.S., Cho, S.H., Lee, J.H., Ahn, G.W., Kim, J.P., Kang, S.M., and Kim, E.K., Ann. Dermatol., 2016, vol. 28, no. 5, p. 555 .

https://doi.org/10.5021/ad.2016.28.5.555

16. Lachowicz, J.I., Nurchi, V.M., Crisponi, G., Pelaez, M.D.G.J., Rescigno, A., Stefanowicz, P., Cal, M., and Szewczuk, Z., J. Inorg. Biochem., 2015, vol. 151, p. 36

17. Hashemi, S.M. and Emami, S., Pharm. Biomed. Res., 2015, vol. 1, no. 1, p. 1.

https://doi.org/10.18869/acadpub.pbr.1.1.1

18. Karakaya, G., Türe, A., Ercan, A., Öncül, S., and Aytemir, M.D., Bioorg. Chem., 2019, vol. 88, paper 102950.

https://doi.org/10.1016/j.bioorg.2019.102950

19. Ashooriha, M., Khoshneviszadeh, M., Khoshneviszadeh, M., Moradi, S.E., Rafiei, A., Kardan, M., and Emami, S., Bioorg. Chem., 2019, vol. 82, p. 414.

https://doi.org/10.1016/j.bioorg.2018.10.069

20. Ashooriha, M., Khoshneviszadeh, M., Khoshneviszadeh, M., Rafiei, A., Kardan, M., Yazdian-Robati, R., and Emami, S., Eur. J. Med. Chem., 2020, vol. 201, Paper 112480.

https://doi.org/10.1016/j.ejmech.2020.112480

21. Rho, H.S., Baek, H.S., Ahn, S.M., Kim, M.K., Ghimeray, A.K., Cho, D.H., and Hwang, J.S., Bull. Korean
Chem. Soc., 2010, vol. 31, no. 8, p. 2375. https://doi.org/10.5012/bkcs.2010.31.8.2375

22. Xie, W., Zhang, J., Ma, X., Yang, W., Zhou, Y., Tang, X., Zou, Y., Li, H., He, J., Xie, S., Zhao, Y., and Liu, F., Chem. Biol. Drug Des., 2015, vol. 86, no. 5, p. 1087. https://doi.org/10.1111/cbdd.12577

23. Takeuchi, K., Hattori, Y., Kawabata, S., Futamura, G., Hiramatsu, R., Wanibuchi, M., Tanaka, H., Masunaga, S-i., Ono, K., Miyatake, S.-I., and Kirihata, M., Cells, 2020, vol. 9, no. 6, Paper N 1551. https://doi.org/10.3390/cells9061551

24. Maloney, P.R., Khan, P., Hedrick, M., Gosalia, P., Milewski, M., Li, L., Roth, G.P., Sergienko, E., Suyama, E., Sugarman, E., Nguyen, K., Mehta, A., Vasile, S., Su, Y., Stonich, D., Nguyen, H., Zeng, F.-Y., Novo, A.M., Vicchiarelli, M., Diwan, J., Chung, T.D.Y., Smith, L.H., and Pinkerton, A.B., Bioorg. Med. Chem. Lett., 2012, vol. 22, no. 21, p. 6656.

https://doi.org/10.1016/j.bmcl.2012.08.105

25. Lucas, S.D., Gonçalves, L.M., Carvalho, L.A.R., Correia, H.F., Da Costa, E.M.R., Guedes, R.A., Moreira, R., and Guedes, R.C., J. Med. Chem., 2013, vol. 56 , no. 23 , p. 9802. https://doi.org/10.1021/jm4011725

26. Karakaya, G., Aytemir, M.D., Özçelik, B., and Çalış, Ü., J. Enzyme Inhib. Med. Chem., 2013, vol. 28, no. 3, p. 627. https://doi.org/10.3109/14756366.2012.666538

27. Karakaya, G., Ercan, A., Öncül, S., and Aytemir, M.D., J. Res. Pharm., 2019, vol. 23, no. 4, p. 596. https://doi.org/10.12991/jrp.2019.167

28. Krimmel, C., Patent US 2700045, 1955; C. A., 1956, vol. 50. 411.

29. Veverka, M., Chem. Pap., 1992, vol. 46, no. 3, p. 208.

30. Kipnis, F., Soloway, H., and Ornfelt, J., J. Am. Chem. Soc., 1948, vol. 70, no. 12, p. 4264. https://doi.org/10.1021/ja01192a080

31. Krimmel, C., Patent US 2715130, 1955.

32. Krimmel C., Patent US 2851467, 1958.

33. White, R.L., Jr., Schwan, T.J., and Alaimo, R.J., J. Heterocycl. Chem., 1980, vol. 17, no. 4, p. 817. https://doi.org/10.1002/jhet.5570170442

34. Rho, H.S., Ahn, S.M., Yoo, D.S., Kim, M.K., Cho, D.H., and Cho, J.Y., Bioorg. Med. Chem. Lett., 2010, vol. 20, no. 22, p. 6569. https://doi.org/10.1016/j.bmcl.2010.09.042

35. Rho, H.S., Yoo, D.S., Ahn, S.M., Kim, M.K., Cho, D.H., and Cho, J.Y., Bull. Korean Chem. 
Soc., 2010, vol. 31, no. 11, p. 3463.

https://doi.org/10.5012/bkcs.2010.31.11.3463

36. Uher, M., Kyselicova, L., Rajniakova, O., Hudecova, D., Bransova, J., and Brtko, J., Chem. Pap., 1997, vol. 51, no. $6 \mathrm{~B}$, p. 421.

37. Rondahl, L., Uher, M., and Brtko, J., Heterocycl. Commun., 2003, vol. 9, no. 3, p. 257.

https://doi.org/10.1515/HC.2003.9.3.257

38. Uher, M., Szymońska, J., Korenova, A., and Tomasik, P., Monatsh. Chem., 2000, vol. 131, no. 3, p. 301. https://doi.org/10.1007/s007060070106

39. Bransova, J., Uher, M., Novotny, L., and Brtko, J., Anticancer Res., 1997, vol. 17, p. 1175.

40. Wu, Z., Cao, A., Ding, W., Zhu, T., and Shen, P., J. Carbohydr. Chem., 2016, vol. 35, no. 7, p. 355. https://doi.org/10.1080/07328303.2016.1261881

41. Xie, W., Zhang, H., He, J., Zhang, J., Yu, Q., Luo, C., and Li, S., Bioorg. Med. Chem. Lett., 2017, vol. 27, no. 3 , p. 530. https://doi.org/10.1016/j.bmcl.2016.12.027

42. Raje, M., Hin, N., Duvall, B., Ferraris, D.V., Berry, J.F., Thomas, A.G., Alt, J., Rojas, C., Slusher, B.S., and Tsukamoto, T., Bioorg. Med. Chem. Lett., 2013, vol. 23, no. 13, p. 3910. https://doi.org/10.1016/j.bmcl.2013.04.062

43. Sherafati, M., Mirzazadeh, R., Barzegari, E., Mohammadi-Khanaposhtani, M., Azizian, H., Asgari, M.S., Hosseini, S., Zabihi, E., Mojtabavi, S., Faramarzi, M.A., Mahdavi, M., Larijani, B., Rastegar, H., Hamedifar, H., and Hajimiri, M.H., Bioorg. Chem., 2021, vol. 109, Paper 104703.

https://doi.org/10.1016/j.bioorg.2021.104703

44. Sepehri, N., Iraji, A., Yavari, A., Asgari, M.S., Zamani, S., Hosseini, S., Bahadorikhalili, S., Pirhadi, S., Larijani, B., Khoshneviszadeh, M., Hamedifar, H., Mahdavi, M., and Khoshneviszadeh, M., Bioorg. Med. Chem., 2021, vol. 36, Paper 116044. https://doi.org/10.1016/j.bmc.2021.116044

45. Schrader, G., Lorenz, W., Cölin, R., and Schlör, H.-H., Patent US 3232830, 1966; C. A., 1966, vol. 64. 15923.

46. Metivier, J., Patent US 2752283, 1956.

47. Litvinov, V.P., Rodinovskaya, L.A., Sharanin, Yu.A., Shestopalov, A.M., and Senning, A., J. Sulfur Chem., 1992, vol. 13, no. 1, p. 1. https://doi.org/10.1080/01961779208048951

48. Litvinov, V.P., Phosphorus, Sulfur, Silicon, Relat. Elem., 1993, vol. 74, no. 1, p. 139. https://doi.org/10.1080/10426509308038105
49. Litvinov, V.P., Russ. Chem. Bull., 1998, vol. 47, no. 11, p. 2053.

https://doi.org/10.1007/BF02494257

50. Litvinov, V.P., Krivokolysko, S.G., and Dyachenko, V.D., Chem. Heterocycl. Compd., 1999, vol. 35, no. 5, p. 509. https://doi.org/10.1007/BF02324634

51. Litvinov, V.P., Dotsenko, V.V., and Krivokolysko, S.G., Russ. Chem. Bull., 2005, vol. 54, no. 4, p. 864.

https://doi.org/10.1007/s11172-005-0333-1

52. Litvinov, V.P., Russ. Chem. Rev., 2006, vol. 75, no. 7 , p. 577. https://doi.org/10.1070/RC2006v075n07ABEH003619

53. Litvinov, V.P., Dotsenko, V.V., and Krivokolysko, S.G., Adv. Heterocycl. Chem., 2007, vol. 93, p. 117. https://doi.org/10.1016/S0065-2725(06)93003-7

54. Dotsenko, V.V., Buryi, D.S., Lukina, D.Yu., and Krivokolysko, S.G., Russ. Chem. Bull., 2020, vol. 69 , no. 10, p. 1829. https://doi.org/10.1007/s11172-020-2969-2

55. Salem, M.A., Helel, M.H., Gouda, M.A., Ammar, Y.A., and El-Gaby, M.S.A., Synth. Commun., 2018, vol. 48, no. 4 , p. 345 .

https://doi.org/10.1080/00397911.2017.1394468

56. Gouda, M.A., Attia, E., Helal, M.H., and Salem, M.A., J. Heterocycl. Chem., 2018, vol. 55, no. 10, p. 2224. https://doi.org/10.1002/jhet.3298

57. Gouda, M.A., Hussein, B.H., Helal, M.H., and Salem, M.A., J. Heterocycl. Chem., 2018, vol. 55, no. 7, p. 1524.

https://doi.org/10.1002/jhet.3188

58. Shamroukh, A.H., Kotb, E.R., Anwar, M.M., and Sharaf, M., Egypt. J. Chem., 2021, vol. 64, no. 8, p. 4509. https://doi.org/10.21608/EJCHEM.2021.64971.3392

59. Quiliano, M. and Aldana, I., Rev. Virtual Quim., 2013, vol. 5 , no. 6 , p. 1120. https://doi.org/10.5935/1984-6835.20130081

60. Mamedov, V.A. and Zhukova, N.A., Progress Heterocycl. Chem., 2012, vol. 24, p. 55.

https://doi.org/10.1016/B978-0-08-096807-0.00002-6

61. Mamedov, V.A. and Zhukova, N.A., Progress Heterocycl. Chem., 2013, vol. 25, p. 1. https://doi.org/10.1016/B978-0-08-099406-2.00001-7

62. Cheng, G., Sa, W., Cao, C., Guo, L., Hao, H., Liu, Z., Wang, X., and Yuan, Z., Front. Pharmacol., 2016, vol. 7, Paper 64.

https://doi.org/10.3389/fphar.2016.00064 
63. González, M. and Cerecetto, H., Exp. Opin. Therap. Pat., 2012, vol. 22, no. 11, p. 1289. https://doi.org/10.1517/13543776.2012.724677

64. Pereira, J.A., Pessoa, A.M., Cordeiro, M.N.D.S., Fernandes, R., Prudêncio, C., Noronha, J.P., and Vieira, M., Eur. J. Med. Chem., 2015, vol. 97, p. 664. https://doi.org/10.1016/j.ejmech.2014.06.058

65. Montana, M., Mathias, F., Terme, T., and Vanelle, P., Eur. J. Med. Chem., 2019, vol. 163, p. 136. https://doi.org/10.1016/j.ejmech.2018.11.059

66. Ajani, O.O., Eur. J. Med. Chem., 2014, vol. 85, p. 688. https://doi.org/10.1016/j.ejmech.2014.08.034

67. El Newahie, A.M.S., Ismail, N.S.M., Abou El Ella, D.A., and Abouzid, K.A.M., Arch. Pharm., 2016, vol. 349 , no. 5 , p. 309 . https://doi.org/10.1002/ardp.201500468

68. Lipinski, C.A., Lombardo, F., Dominy, B.W., and Feeney, P.J., Adv. Drug. Delivery Rev., 1997, vol. 23, nos. $1-3$, p. 4. https://doi.org/10.1016/S0169-409X(96)00423-1

69. Lipinski, C.A., Drug Discov. Today: Technologies, 2004, vol. 1, no. 4, p. 337. https://doi.org/10.1016/j.ddtec.2004.11.007

70. Lipinski, C.A., Lombardo, F., Dominy, B.W., and Feeney, P.J., Adv. Drug. Delivery Rev., 2012, vol. 64, Suppl, p. 4. https://doi.org/10.1016/j.addr.2012.09.019

71. Sander, T., OSIRIS Property Explorer. Idorsia Pharmaceuticals Ltd, Switzerland. http://www.organic-chemistry.org/prog/peo/

72. Daina, A., Michielin, O., and Zoete, V., Sci. Rep., 2017, vol. 7, Article N 42717. https://doi.org/10.1038/srep42717

73. Lagunin, A., Zakharov, A., Filimonov, D., and Poroikov, V., Mol. Inform., 2011, vol. 30, nos. 2-3, p. 241. https://doi.org/10.1002/minf.201000151

74. OECD Environment, Health and Safety Publications Series on Testing and Assessment no. 24. Guidance Document on Acute oral Toxicity Test- ing. ENV/JM/MONO(2001)4. OECD, Paris. https:/www.oecd.org/officialdocuments/publicdisplaydo cumentpdf $/$ ?cote $=$ env $/ \mathrm{jm} / \mathrm{mono}(2001) 4 \&$ doclanguage $=$ en

75. Yang, J., Kwon, S., Bae, S.H., Park, K.M., Yoon, C., Lee, J.H., and Seok, C., J. Chem. Inf. Model., 2020, vol. 60, no. 6, p. 3246. https://doi.org/10.1021/acs.jcim.0c00104

76. GalaxyWEB. A web server for protein structure prediction, refinement, and related methods. Computational Biology Lab, Department of Chemistry, Seoul National University, S. Korea. http://galaxy.seoklab.org/index.html

77. Ko, J., Park, H., Heo, L., and Seok, C., Nucleic Acids Res., 2012, vol. 40, no. W1, p. W294. https://doi.org/10.1093/nar/gks493

78. Pettersen, E.F., Goddard, T.D., Huang, C.C., Couch, G.S., Greenblatt, D.M., Meng, E.C., and Ferrin, T.E., J. Comput. Chem. 2004, vol. 25, no. 13, p. 1605. https://doi.org/10.1002/jcc.20084

79. UCSF Chimera. Visualization system for exploratory research and analysis developed by the Resource for Biocomputing, Visualization, and Informatics at the University of California, San Francisco, US. https://www.rbvi.ucsf.edu/chimera/

80. Buryi, D.S., Dotsenko, V.V., Levashov, A.S., Lukina, D.Yu., Strelkov, V.D., Aksenov, N.A., Aksenova, I.V., and Netreba, E.E., Russ. J. Gen. Chem., 2019, vol. 89, no. 5, p. 886. https://doi.org/10.1134/S1070363219050050

81. Shestopalov, A.M., Nikishin, K.G., Gromova, A.V., and Rodinovskaya, L.A., Russ. Chem. Bull., 2003, vol. 52, no. 10 , p. 2203. https://doi.org/10.1023/B:RUCB.0000011879.89900.1f

82. Viola, H., Mayer, R., and Jähne, E., Patent DD 144917 , 1980.

83. Aghbash, K.O., Pesyan, N.N., Marandi, G., Dege, N., and Şahin, E., Res. Chem. Intermed., 2019, vol. 45, no. 9 , p. 4543.

https://doi.org/10.1007/s11164-019-03848-7 